\title{
Searching for X-ray Binaries in the LMC: The Optical Counterparts of CAL 9 and CAL E
}

\author{
PAUL C. SCHMIDTKE \& ANNE P. COWLEY \\ Department of Physics and Astronomy, Arizona State University, \\ Tempe, AZ 85287-1504, USA
}

\section{INTRODUCTION}

To better define the global properties of X-ray binaries in the Large Magellanic Cloud, a program of optical identification has been initiated, combining improved X-ray positions from ROSAT with photometry and spectroscopy obtained at CTIO. This initiative is an extension of work by Cowley et al. (1984) and Crampton et al. (1985), who attempted to identify the optical counterparts of "CAL" X-ray point sources found in the Einstein IPC survey of the LMC conducted by Columbia Astrophysical Laboratory (Long et al. 1981). A key element in our investigation of the remaining unidentified sources is the use of significantly more accurate coordinates (typically $\pm 5^{\prime \prime}$ from ROSAT HRI data versus $\pm 30^{\prime \prime}$ for the older IPC positions), thereby greatly reducing the number of stars needing examination. Likely candidates are selected using multicolor photometry (by location in color-color plots or through variability) prior to spectroscopic observing. Although the identifications for CAL 9 and CAL E were reasonably secure (in particular the latter since it had an Einstein HRI position, see Crampton et al. ), these sources have been re-observed to test our procedures and to verify the previous results.

\section{OBSERVATIONS}

ROSAT HRI observations of CAL 9 and CAL E were obtained in Feb. 1991, and coordinates for these sources were determined by standard pipeline processing of the X-ray data. CCD images of the surrounding fields were obtained in Nov./Dec. 1991 and Feb. 1992 with the CTIO 0.9m telescope. The astrometry of each CCD field was calibrated using secondary position standards, which are tied to SAO stars measured on a glass copy of the ESO/ERC sky survey. $V$ filter images of the CAL 9 and CAL E fields are shown in Figures 1 and 2 , respectively, with the ROSAT positions indicated by small circles (arbitrarily drawn with $5^{\prime \prime}$ radii). In each figure the brightest star near the predicted position is the likely optical counterpart.

\subsection{CAL 9}

Differential photometry of the suggested candidate for CAL 9 has been calculated relative to other bright stars in the field. The stellar image of the optical counterpart is a blend that contains two faint companions. One contaminant is $\sim 2^{\prime \prime}$ east and $\sim 2 \mathrm{mag}$ fainter (in $V$ ) while the second is $\sim 4^{\prime \prime}$ north and $\sim 4$ mag fainter. Subtracting the contribution from these stars, the light and color curves for the candidate itself are exceptionally flat (see Figure 1), with $V=$ $14.46 \pm 0.02$ and $B-V=-0.07 \pm 0.01$, which is surprising since the position of 

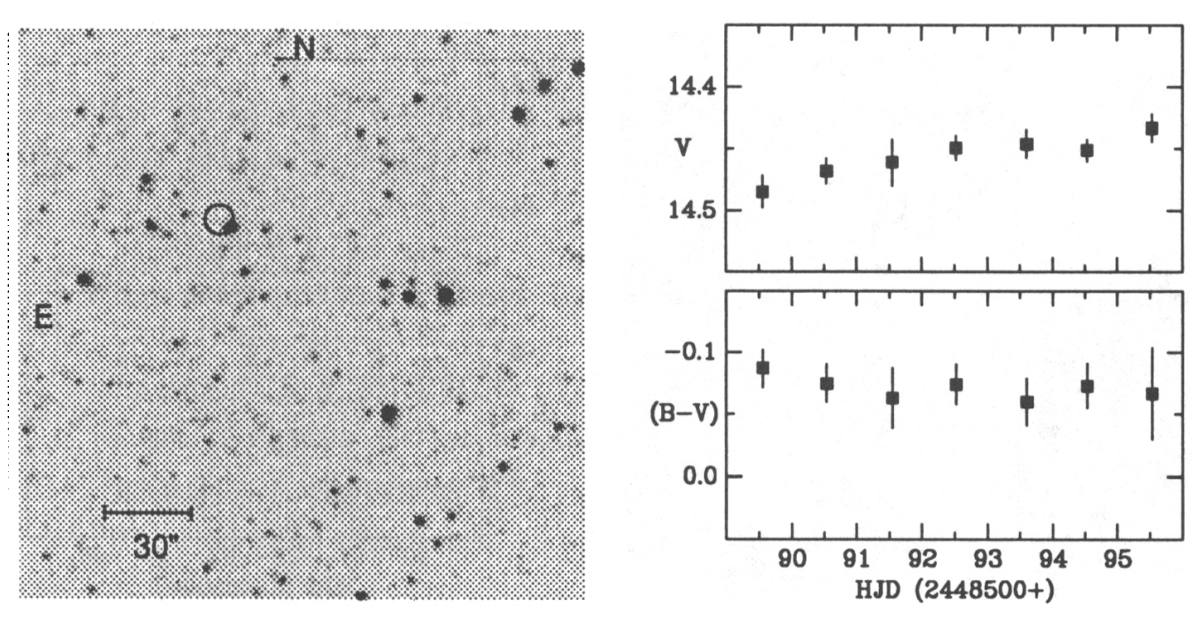

FIGURE 1. CCD image, light curve, and color curve for CAL 9.

CAL 9 coincides with the known variable star HV2289 (amplitude $\sim 0.8 \mathrm{mag}$ ). Although our recent data lacks photometric variabilty that is often present in Xray binaries (e.g., eclipses, ellipsoidal variations, flickering, etc. ), spectroscopy obtained in Feb. 1992 with the CTIO $4 \mathrm{~m}$ telescope reveals variable $\mathrm{H}$ emission, compared to Crampton et al. (1985), and weak absorption lines of H, He I, and He II, suggesting the presence of an additional continuum source - perhaps an accretion disk. Since the optical neighbors cannot account for these spectral signatures, CAL 9 appears to be an excellent candidate for an X-ray binary system. The observed radial velocity range is $>80 \mathrm{~km} \mathrm{~s}^{-1}$, which is consistent with an orbital motion interpretation.

\subsection{CAL E}

The light and color curves of the optical counterpart for CAL $\mathbf{E}$ are shown in Figure 2. Again, they are very flat, having $V=14.24 \pm 0.02$ and $B-V=-0.10$ \pm 0.01 . The ROSAT position coincides with the northern component of a pair of $14^{\text {th }}$ magnitude stars that are separated by $\sim 15^{\prime \prime}$, in agreement with the results of Crampton et al. (1985). Note that two entries in their Table I are reversed; the northern component is the BOIII(e) star while the southern is a Ba II star. Our recent spectroscopy confirms that the northern star has an early spectral type, with a small range of radial velocities $\left(\sim 20 \mathrm{~km} \mathrm{~s}^{-1}\right)$. The spectrum is similar to the optical candidate for CAL 9.

\section{DISCUSSION}

The optical counterparts for CAL 9 and CAL $\mathrm{E}$ are unusual stars. Although they have colors and magnitudes appropriate for late-B supergiants at the distance of the LMC, their spectra indicate much earlier types. The observations are consistent with $\mathrm{O} / \mathrm{B}$ stars that are veiled by light from cool accretion disks, sim- 

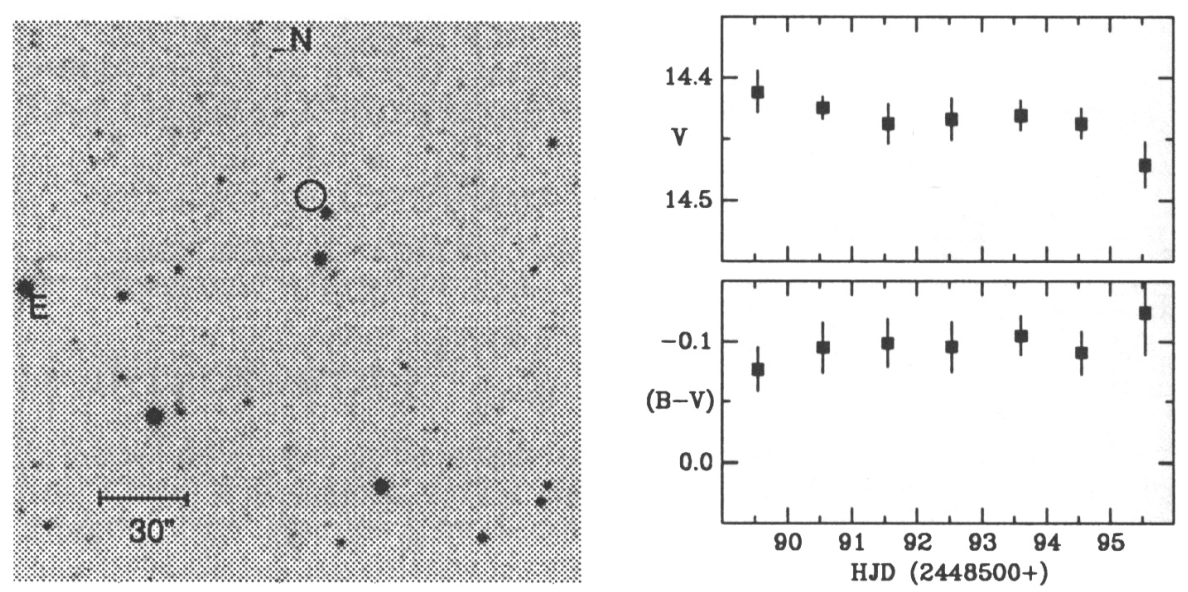

FIGURE 2. CCD image, light curve, and color curve for CAL E.

ilar to the situation present in LMC X-3. In that system, ellipsoidal variations are superposed on a long-term ( $\sim 200$ day) variability in which the composite color becomes redder as the contribution of light originating in the disk increases (Cowley et al. 1991). If CAL 9 and CAL E are high-mass X-ray binaries, then the lack of photometric variability is difficult to explain. For example, massive secondary stars should introduce ellipsoidal variations into the light curves, but this signal has not yet been detected. Possibly, the amplitude is small because the mass ratio, $\mathcal{M}_{\mathbf{X}} / \mathcal{M}_{\text {opt }}$, is small or the physical separation of the binary is large. Not only would the ellipsoidal variations be weak but also the period could be long, further complicating detection. Although low orbital inclination can account for a small amplitude, the range of radial velocities for CAL 9 implies at least a modest inclination. A veiled spectrum also implies that the observed amplitude of ellipsoidal variations is reduced by light coming from another source like an accretion disk, but the disk contribution cannot be so large as to obliterate spectral signatures of the primary star. Clearly, further study of both CAL 9 and CAL $\mathrm{E}$ is needed.

\section{REFERENCES}

Cowley, A.P., Crampton, D., Hutchings, J.B., Helfand, D.J., Hamilton, T.T., Thorstensen, J.R., \& Charles, P.A. 1984, $A p J, 286,196$

Cowley, A.P., Schmidtke, P.C., Ebisawa, K., Makino, F., Remillard, R.A., Crampton, D., Hutchings, J.B., Kitamoto, S., \& Treves, A. 1991, ApJ, 381, 526

Crampton, D., Cowley, A.P., Thompson, I.B., \& Hutchings, J.B. 1985, AJ, 90, 43

Long, K.S., Helfand, D.J., \& Grabelsky, D.A. 1981, ApJ, 248, 925 